\title{
Acetazolamide-Triggered Acute Flaccid Paralysis and Toxic-Metabolic Encephalopathy in a Toddler with Gastroenteritis: A Case Report
}

\author{
Jack Green ${ }^{1}$ Pooja Nawathe ${ }^{1}$ \\ ${ }^{1}$ Division of Pediatric Critical Care, Department of Pediatrics, Cedars- \\ Sinai Medical Center, Los Angeles, California, United States \\ J Child Sci 2020;10:e159-e162.
}

\begin{abstract}
Address for correspondence Jack Green, MD, Division of Pediatric Critical Care, Department of Pediatrics, Cedars-Sinai Medical Center, 8700 Beverly Blvd, NT 4255B, Los Angeles, CA 90048, United States (e-mail: jack.green@cshs.org).
\end{abstract}

\begin{abstract}
Keywords

- encephalopathy

- metabolic derangement

- ketotic hypoglycemia

- dehydration

Acetazolamide is an infrequently prescribed medication in the outpatient pediatric setting, as one of its major indications is for the treatment of glaucoma, which is a largely adult disease. Though incredibly rare, serious neurologic side effects such as stroke, flaccid paralysis, and coma can occur after its administration. This case presentation of acute flaccid paralysis with metabolic derangement underscores the unusual, yet possible, toxic-metabolic and neurologic sequelae that can occur in an already acidotic host (in this case, caused by gastroenteritis) with acetazolamide ingestion. Life-threatening conditions must always be ruled out in patients who present with encephalopathy, but a medication history was crucial in clinching this case's unifying diagnosis. While there are case reports highlighting the rare central nervous system toxicities associated with acetazolamide ingestion, to our knowledge, none exist in the pediatric literature.
\end{abstract}

\section{Introduction}

Acetazolamide is an infrequently prescribed medication in the outpatient pediatric setting, but it is commonly used in the critical care setting. ${ }^{1}$ Its intended effects on the acid-base balance can lead to significant pathology in an already ill patient. $^{2}$ A broad differential must always be used in any patient presenting with encephalopathy, ${ }^{3}$ but an environmental and medication history is equally important in a patient's final assessment. Though there are adult case reports on the rare effects of acetazolamide on the central nervous system (CNS), ${ }^{4,5}$ here we report the first pediatric case, with acetazolamide being implicated in the development of paralysis in a toddler.

\section{Case Report}

The patient is a 3-year-old female of Japanese descent with congenital glaucoma, chromosomal microdeletion (16q23.1), and mild developmental delay, who was in her usual state of health until she developed vomiting and diarrhea 4 days before admission. The parents confirm symptoms of gastroenteritis (primarily diarrhea) in several family members at home, with no sick contacts at daycare. They denied fevers, respiratory symptoms, recent consumption of strange foods, recent international travel, unusual pets, or unguarded medicines in the home over the last 2 weeks. The patient is on long-standing dorzolamide-timolol (Cosopt, Akorn Inc., 2-0.5\%, one drop in both eyes twice a day) and travoprost (Travatan Z, Novartis Inc., $0.004 \%$, one drop in both eyes nightly) ophthalmic drops for her congenital glaucoma with the addition of acetazolamide (Diamox, Mutual Inc., $125 \mathrm{mg}$ three times a day by mouth, 30 $\mathrm{mg} / \mathrm{kg} /$ day) 4 days before her gastrointestinal symptoms began.

Eight days after the initiation of acetazolamide, the patient presented to the emergency room (ER) in a quaternary care institution in California, United States. Fully conversive and interactive the night before the presentation, the parents received

July 1,2020

accepted

September 8, 2020
DOI https://doi.org/

10.1055/s-0040-1718562. ISSN 2474-5871.
Copyright (c) 2020 Georg Thieme Verlag License terms KG Stuttgart · New York
(1) (1) 
found her minimally responsive and unable to move her arms or legs in the morning. They attempted to arouse her but without success; therefore, she is immediately brought to the ER for altered mental status. Initial vitals were as follows: a heart rate of 80 beats per minute, respiratory rate of 26 breaths per minute, an oral temperature of $36.3^{\circ} \mathrm{C}$, blood pressure of $103 / 62 \mathrm{~mm} \mathrm{Hg}$, and an oxygen saturation level of $98 \%$ on room air. She was found to be catatonic with worrisome examination findings of areflexia in both upper and lower extremities, as well as intermittent anisocoria. Pupils were still reactive, she had a gag reflex, but was flaccid below the level of the neck. The rest of her examination (cardiovascular, respiratory, gastrointestinal) was unrevealing. Initial laboratory findings revealed severe hypoglycemia $(<20 \mathrm{mg} / \mathrm{dL}$ ), severe metabolic acidosis (serum bicarbonate of $11 \mathrm{mmol} / \mathrm{L}$, venous $\mathrm{pH}$ of 7.1), and 4+ calcium hyperoxaluria (see - Table 1 for expanded laboratory results). Seizurelike movements and automatisms lead to the immediate administration of intravenous (IV) dextrose (100 mL of dextrose $10 \%$ in water, $\sim 6 \mathrm{~mL} / \mathrm{kg}$ ) and IV lorazepam (1 mg, $0.06 \mathrm{mg} / \mathrm{kg}$ ). A computed tomography (CT) of the brain was then emergently completed, and a robust infectious and advanced imaging workup was initiated due to the concern for flaccid paralysis. Due to persistent altered mental status and acute flaccid paralysis, she was then admitted to the pediatric intensive care unit (PICU).

Lumbar puncture (LP) with infectious markers including meningoencephalitis (ME) polymerase chain reaction (PCR) panel, toxicology screen, CT of the brain/cervical spine, magnetic resonance imaging (MRI) of the brain/cervical/lumbar/ thoracic spine, and continuous video-EEG (electroencephalogram) monitoring are subsequently completed. Her opening pressure on the LP was $15 \mathrm{~cm} \mathrm{H}_{2} 0$, with no evidence of inflammation or infection on cerebrospinal fluid studies.

MRI did demonstrate slight enhancement of the cauda equina and small prominence of the lumbar nerve roots, but it was thought to be of no clinical significance. ME PCR panel was negative for typical pathogens causing ME, toxicology screen was negative, and video-EEG showed no seizure activity. The patient's stool studies (including Shigella, Shiga toxin, Campylobacter, Salmonella, Clostridium difficile, Giardia, Cryptosporidium, Entamoeba, and Rotavirus) were negative. Despite no respiratory symptoms, an expanded respiratory viral PCR panel was subsequently sent. The panel was positive for rhino/enterovirus, parainfluenza 4 , and respiratory syncytial virus (RSV).

Within 6 hours of admission to the PICU, the patient began to have purposeful movements after correction of her hypoglycemia and severe acidosis with rehydration, glucose, and supplemental bicarbonate administration. Within 24 hours, she was completely back to her neurologic baseline. Broadspectrum meningitic dosing of IV ceftriaxone, IV vancomycin, and IV acyclovir was discontinued after 48 hours, with final cultures and HSV (herpes simplex virus) cerebrospinal fluid (CSF) PCR yielding negative results. Video-EEG did not show epileptiform discharges and was discontinued after the patient's mental status returned to baseline. She began to eat, drink, and ambulate with assistance and was discharged after a short 2-day intensive care unit admission. Two days after
Table 1 Initial laboratory testing completed for toddler presenting with acute encephalopathy

\begin{tabular}{|c|c|c|}
\hline Laboratory test & Result & Reference range \\
\hline $\begin{array}{l}\text { White blood cell } \\
\text { count }(1,000 / \mathrm{UL})\end{array}$ & 13.95 & $4.86-13.18$ \\
\hline Hemoglobin (g/dL) & 14.7 & $10.2-12.7$ \\
\hline Hematocrit (\%) & 46.8 & $31.2-37.8$ \\
\hline Platelets (1,000/UL) & 349 & $150-450$ \\
\hline Neutrophils (\%) & 76.1 & $\mathrm{~N} / \mathrm{A}$ \\
\hline Lymphocytes (\%) & 21.1 & $\mathrm{~N} / \mathrm{A}$ \\
\hline Eosinophils (\%) & 0.1 & $\mathrm{~N} / \mathrm{A}$ \\
\hline $\mathrm{pH}$ venous & 7.16 & $7.30-7.40$ \\
\hline $\mathrm{pCO}_{2}$ venous $(\mathrm{mm} \mathrm{Hg})$ & 32 & $40-50$ \\
\hline $\mathrm{pO}_{2}$ venous $(\mathrm{mm} \mathrm{Hg})$ & 76 & $35-45$ \\
\hline $\mathrm{HCO}_{3}$ venous (mmol/L) & 11.4 & $23-29$ \\
\hline $\begin{array}{l}\text { Base excess } \\
\text { venous }(\mathrm{mmol} / \mathrm{L})\end{array}$ & -17 & -2 to +2 \\
\hline Lactate (mmol/L) & 1.3 & $0.5-2.2$ \\
\hline Urine ketones & $2+$ & $\mathrm{N} / \mathrm{A}$ \\
\hline Glucose $(\mathrm{mg} / \mathrm{dL})$ & $<20$ & $70-99$ \\
\hline Sodium (mmol/L) & 136 & $135-145$ \\
\hline Potassium (mmol/L) & 3.7 & $3.5-5.0$ \\
\hline Chloride (mmol/L) & 105 & $98-107$ \\
\hline Carbon dioxide (mmol/L) & 11 & $22-31$ \\
\hline Anion gap (mEq/L) & 24 & $10-20$ \\
\hline Urea nitrogen (mg/dL) & 29 & $5.1-16.8$ \\
\hline Creatinine (mg/dL) & 0.5 & $0.39-0.55$ \\
\hline C-reactive protein (mg/L) & $<0.2$ & $<5$ \\
\hline $\begin{array}{l}\text { Sedimentation } \\
\text { rate (mm/hour) }\end{array}$ & 7 & $<20$ \\
\hline CSF RBC count (/UL) & 0 & $0-25$ \\
\hline CSF WBC count (/UL) & 1 & $0-20$ \\
\hline CSF PMN (\%) & 0 & $\mathrm{~N} / \mathrm{A}$ \\
\hline CSF lymphocytes (\%) & 2 & $\mathrm{~N} / \mathrm{A}$ \\
\hline CSF glucose $(\mathrm{mg} / \mathrm{dL})$ & 24 & $45-80$ \\
\hline CSF protein $(\mathrm{mg} / \mathrm{dL})$ & 27 & $15-45$ \\
\hline
\end{tabular}

Note: Bolded values represent abnormal labs.

Abbreviations: CSF, cerebrospinal fluid; N/A, not applicable; PMN, polymorphonuclear leukocyte; RBC, red blood cell; WBC, white blood cell.

discharge from the hospital, the patient had a follow-up with her pediatrician, who noted that she was doing remarkably well, reporting a normal mental status and regular activity. The ophthalmologist with the family decided to pursue surgery for her glaucoma rather than reintroduce acetazolamide.

\section{Discussion}

Life-threatening conditions must always be ruled out in patients who present with encephalopathy and new-onset neurologic findings, especially flaccid paralysis. ${ }^{3}$ Imminently 
harmful differential diagnoses of status epilepticus, acute transverse myelitis, infection-mediated Guillain-Barré's syndrome, and acute demyelinating encephalomyelitis were all considered and ruled out during the patient's work-up. ${ }^{6-9}$

With the patient's gastrointestinal symptoms, rotavirusassociated CNS manifestations were also considered. ${ }^{10}$ Possible sequelae, including both encephalopathy and flaccid paralysis, present in our case have been reported. ${ }^{11}$ Though its mechanism is unclear, transient viral toxicity in the CNS may be implicated in its pathophysiology. ${ }^{12}$ As the patient's stool studies were all negative, this diagnosis was unlikely. Though inflammatory markers were low with LP findings unrevealing for CNS disease, infection continued to remain high on our differential. Enterovirus-associated (EV-D68) acute flaccid myelitis cases gained traction in 2014 when an outbreak caused more than 1,000 cases across the United States with variable neurophenotypic expressivity. ${ }^{13}$ Though our patient tested positive for Enterovirus, she had no respiratory symptoms, no CSF pleocytosis, and no lesions in the anterior horn of the gray matter along the spinal cord or the brainstem of her MRI to suggest EV-D68. ${ }^{14}$ Our patient eventually improved too quickly, lending the ultimate diagnosis to a combination of multifactorial severe ketotic hypoglycemia/dyselectrolytemia compounded by the introduction of a new medicine.

Recently, her ophthalmologist had concerns for worsening intraocular hypertension, which prompted the prescription of acetazolamide as an outpatient (Diamox, Mutual Inc., $125 \mathrm{mg}$ three times a day by mouth, $30 \mathrm{mg} / \mathrm{kg} /$ day ). Acetazolamide is a noncompetitive carbonic anhydrase (CA) inhibitor that is commonly used to treat glaucoma or altitude sickness, and historically used for epilepsy. ${ }^{15}$ In the pediatric critical care environment, it is frequently used to treat iatrogenic metabolic alkalosis in the mechanically ventilated patient. ${ }^{1}$ CA is an enzyme that generally potentiates the redox reaction of $\mathrm{H}_{2} \mathrm{CO}_{3} \rightleftharpoons \mathrm{HCO}_{3}{ }^{-}+\mathrm{H}^{+}$(carbonic acid $\rightleftharpoons$ bicarbonate + hydrogen ion), but its inhibition in the proximal tubule of the kidney leads to an efflux of bicarbonate and the intended consequence of metabolic acidosis. ${ }^{16}$ Its typical side effects include nausea, vomiting, diarrhea, excessive metabolic acidosis, hypokalemia, and hypoglycemia, almost all of which our patient experienced. ${ }^{17}$ Topical CA inhibitors such as dorzolamide, though locally instilled as opposed to systemic oral administration, also work by decreasing the rate of aqueous humor secretion, thereby lowering intraocular pressure. ${ }^{18}$ The rationale of adding oral acetazolamide to topical CA inhibitor drops for the treatment of pediatric glaucoma has been shown in one study to concurrently reduce elevated intraocular pressures, ${ }^{19}$ prompting its initiation in our patient.

This unique presentation of acute flaccid paralysis and encephalopathy underscores the rare, yet possible, toxicmetabolic and neurologic derangements that can occur in an already compromised state (gastroenteritis in our patient) following acetazolamide ingestion, even at appropriate pediatric dosing guidelines. ${ }^{20}$ Though severe hypoglycemia itself can cause variable presentations of paralysis (hemiplegia, tetraplegia, aphasia), ${ }^{21,22}$ we hypothesize that the neurologic derangements seen in our case presentation were likely multifactorial with an initial insult of community- acquired gastroenteritis compounded by known acetazolamide-associated dyselectrolytemia. Ketotic hypoglycemia (as evidenced by a serum blood glucose of $<20$ and urine ketones of $2+$ ) from ongoing gastrointestinal losses was likely the essential trigger that led to the beginning of the patient's clinical manifestations. Our patient also had prerenal azotemia due to dehydration, leading to the decreased elimination of the drug in the body, as the majority of acetazolamide is renally excreted within 24 hours. $^{23}$ This may have contributed to the exaggerated effects at normal dose administration.

Inatani et al reported that acetazolamide concentration in the red blood cells may be related to the occurrence of its side effects. ${ }^{24}$ There are interindividual differences in the absorption lag and peak times of acetazolamide plasma concentrations, with two types of CA isoenzymes showing different affinities for inhibitors in human erythrocytes, and both isoenzymes can be inhibited by the effect of acetazolamide. ${ }^{25}$ Thus, the inhibited activities of CA isoenzymes in erythrocytes by acetazolamide may be another explanation for its more pronounced side effects in patients with exaggerated absorption lag and peak times. Adult case reports highlight the rare CNS toxicities associated with acetazolamide ingestion, ${ }^{4,5}$ such as cerebellar stroke, uremic encephalopathy, delirium, and coma. To our knowledge, no case reports exist in the pediatric literature; hence, our report describes this rare but important complication seen with acetazolamide ingestion.

\section{Conclusion}

Metabolic derangements such as critical ketotic hypoglycemia and acidosis can mimic life-threatening neurologic disease. A detailed environmental exposure history, including recent medication initiation or change in dosing, is crucial for the work-up of acute onset altered mental status. In this case report, the addition of acetazolamide in a child with gastroenteritis and prerenal azotemia likely contributed to the severe presentation of flaccid paralysis. Acetazolamide is not a commonly prescribed medication in the outpatient pediatric setting. Though incredibly rare, significant neurologic side effects such as stroke, coma, and flaccid paralysis can occur after its administration in the context of dehydration, acidosis, and prerenal azotemia.

\section{Disclosure}

Authorization for the use or disclosure of health information for case report publication was signed by the guardian.

\section{Conflict of Interest}

None declared.

\section{References}

1 López C, Alcaraz AJ, Toledo B, Cortejoso L, Gil-Ruiz MA. Acetazolamide therapy for metabolic alkalosis in pediatric intensive care patients. Pediatr Crit Care Med 2016;17(12):e551-e558

2 Heller I, Halevy J, Cohen S, Theodor E. Significant metabolic acidosis induced by acetazolamide. Not a rare complication. Arch Intern Med 1985;145(10):1815-1817 
3 Goto S, Nosaka N, Yorifuji T, et al. Epidemiology of pediatric acute encephalitis/encephalopathy in Japan. Acta Med Okayama 2018; 72(04):351-357

4 Mendes TM. Stroke mimic caused by acetazolamide. Eur J Case Rep Intern Med 2018;5(05):000822

5 Schwenk MH, St Peter WL, Meese MG, Singhal PC. Acetazolamide toxicity and pharmacokinetics in patients receiving hemodialysis. Pharmacotherapy 1995;15(04):522-527

6 Boylan GB, Kharoshankaya L, Mathieson SR. Diagnosis of seizures and encephalopathy using conventional EEG and amplitude integrated EEG. Handb Clin Neurol 2019;162:363-400

7 West TW. Transverse myelitis-a review of the presentation, diagnosis, and initial management. Discov Med 2013;16(88):167-177

8 Illes Z, Blaabjerg M. Cerebrospinal fluid findings in Guillain-Barré syndrome and chronic inflammatory demyelinating polyneuropathies. Handb Clin Neurol 2017;146:125-138

9 Pohl D, Alper G, Van Haren K, et al. Acute disseminated encephalomyelitis: Updates on an inflammatory CNS syndrome. Neurology 2016;87(09, Suppl 2):S38-S45

10 Incecik F, Hergüner MO, Altunbaşak S, Solgun H. Acute encephalopathy associated rotavirus gastroenteritis. J Pediatr Neurosci 2009;4(02):141-143

11 Özalp E, Aydın-Teke T, Tanır G, Özkan M, Bayhan GI. Transient acute flaccid paralysis and seizures associated with rotavirus gastroenteritis in a child. Turk J Pediatr 2012;54(06):661-663

12 Kawamura Y, Ohashi M, Ihira M, Hashimoto S, Taniguchi K, Yoshikawa T. Nationwide survey of rotavirus-associated encephalopathy and sudden unexpected death in Japan. Brain Dev 2014; 36(07):601-607

13 Messacar K, Abzug MJ, Dominguez SR. 2014 outbreak of enterovirus D68 in North America. J Med Virol 2016;88(05):739-745

14 Knoester M, Helfferich J, Poelman R, Van Leer-Buter C, Brouwer OF, Niesters HGM2016 EV-D68 AFM Working Group. Twenty-nine cases of Enterovirus-D68-associated acute flaccid myelitis in
Europe 2016: a case series and epidemiologic overview. Pediatr Infect Dis J 2019;38(01):16-21

15 Filippi L, Bagnoli F, Margollicci M, Zammarchi E, Tronchin M, Rubaltelli FF. Pathogenic mechanism, prophylaxis, and therapy of symptomatic acidosis induced by acetazolamide. J Investig Med 2002;50(02):125-132

16 Lindskog S. Structure and mechanism of carbonic anhydrase. Pharmacol Ther 1997;74(01):1-20

17 Schmickl CN, Owens RL, Orr JE, Edwards BA, Malhotra A. Side effects of acetazolamide: a systematic review and meta-analysis assessing overall risk and dose dependence. BMJ Open Respir Res 2020;7(01):e000557

18 Portellos M, Buckley EG, Freedman SF. Topical versus oral carbonic anhydrase inhibitor therapy for pediatric glaucoma. J AAPOS 1998;2(01):43-47

19 Sabri K, Levin AV. The additive effect of topical dorzolamide and systemic acetazolamide in pediatric glaucoma. J AAPOS 2006;10 (05):464-468

20 Acetazolamide. In: Drug Dosages. Kahl LK, Hughes HK, eds. Harriet Lane Handbook. Philadelphia, PA, USA: Elsevier; 2018

21 Agrawal N, Jamshed N, Aggarwal P, Ekka M. Severe hypoglycemia masquerading as cerebellar stroke. J Family Med Prim Care 2014;3 (04):440-442

22 Duarte J, Perez A, Coria F, Clavería LE, García MA. Hypoglycemia presenting as acute tetraplegia. Stroke 1993;24(01):143

23 Dollery SC. Therapeutic Drugs. London: Churchill Livingstone; 1991

24 Inatani M, Yano I, Tanihara H, Ogura Y, Honda Y, Inui KI. Relationship between acetazolamide blood concentration and its side effects in glaucomatous patients. J Ocul Pharmacol Ther 1999;15 (02):97-105

25 Wistrand PJ, Bååthe P. Inhibition of carbonic anhydrase activity of whole erythrocytes. Acta Pharmacol Toxicol (Copenh) 1968;26 (02):145-168 